\title{
Searches for Higgs and Higgs-like particles at LHCb
}

\author{
Marcin KUCHARCZYK ${ }^{* \dagger}$ \\ University of Milano-Bicocca and Institute of Nuclear Physics PAN, Krakow \\ E-mail: marcin.kucharczyk@cern.ch
}

\begin{abstract}
A set of results related to the new particles searches in $\mathrm{LHCb}$ are reported. Upper limits on the cross section times branching fraction of neutral Higgs boson decaying into two tau leptons have been set in the model independent search and in the Minimal Supersymmetric Model. Many theoretical models predict the existence of the Higgs bosons decaying into heavy long-lived particles. The upper limit on their production cross section has been determined for several combinations of parameters. A measurement of the cross section for the production of $b \bar{b}$ and $c \bar{c}$ pairs based on inclusive final states has been performed in a region of pseudorapidity $2.5<\eta<4.0$ and transverse momentum $p_{T}>5 \mathrm{GeV}$ of the $b$ hadron candidate, resulting in $\sigma(b \bar{b})=7.7 \pm 0.12$ (stat) \pm 0.84 (syst) and $\sigma(c \bar{c})=104.6 \pm 2.7$ (stat) \pm 11.4 (syst). The forward-central $b \bar{b}$ production asymmetry, based on the selection of high $p_{T}$ jet pairs tagged by the presence of a displaced muon with high momentum, has been determined to be $A_{F C}^{b \bar{b}}=(0.5 \pm 0.5$ (stat) \pm 0.5 (syst) $) \%$ and $A_{F C}^{b \bar{b}}\left(M_{b \bar{b}}\right.$ $>100 \mathrm{GeV})=(4.3 \pm 1.7$ (stat) \pm 2.4 (syst) $)$.
\end{abstract}

The European Physical Society Conference on High Energy Physics

18-24 July, 2013

Stockholm, Sweden

\footnotetext{
*Speaker.

'On behalf of the LHCb collaboration.
} 


\section{Introduction}

The evidence of a new particle produced in proton-proton collisions with a mass of about 125 $\mathrm{GeV}$, has been reported recently by ATLAS and CMS collaborations [1].

The LHCb experiment [2], fully instrumented in the pseudorapidity $2<\eta<5$, is able to access the low $p_{T}$ region and may test models with enhanced forward production. Model-independent together with model-dependent (Minimal Supersymmetric Model (MSSM) [3] Higgs) limits on a Higgs boson cross-section times branching fraction into two tau leptons are presented in this report in Sect. 2. There are many theoretical models of new phenomena beyond the Standard Model $(\mathrm{SM})$. Some of them $[4,5]$ predict massive long-lived particles which can be produced in the Higgs boson decay. Due to the long length of the LHCb vertex detector (VELO) and its excellent tracking capabilities, the LHCb experiment is sensitive to such exotic particles. Preliminary limits on their production cross sections are presented in Sect. 3.

The measurement of the inclusive $b$ and $c$ production rates from QCD processes is crucial to understand the background in searches for massive particles decaying into $b$ quarks, such as the Higgs boson or new heavy particles. The preliminary cross section measurement is presented in Sect. 4. Finally, in Sect. 5, the preliminary measurement of the forward-central asymmetry in the $b \bar{b}$ production is described. It may provide useful information to constrain the models which attempt to understand the top-quark forward-backward production asymmetry [6].

\section{Limits on neutral Higgs boson production in the forward region}

Limits on the production of a neutral Higgs boson decaying into two tau leptons in the forward region are reported based on data collected by LHCb in 2011 at a centre-of-mass energy $\sqrt{s}=7 \mathrm{TeV}$, corresponding to an integrated luminosity of about $1.0 \mathrm{fb}^{-1}$ [7]. Candidates have been identified by reconstructing five categories of exclusive final states: $\tau_{\mu} \tau_{\mu}, \tau_{\mu} \tau_{e}, \tau_{e} \tau_{\mu}, \tau_{\mu} \tau_{h}$ and $\tau_{e} \tau_{h}$, where the subscripts denote muon $(\mu)$, electron ( $e$ ) and hadron $(h)$. The selection is based on high $p_{T}$ leptons, where the $p_{T}$ of the first tau decay product has to be above $20 \mathrm{GeV}$, while for the second particle above $5 \mathrm{GeV}$. Each pair of tau decay products must be approximately back-to-back and each track must be displaced from the primary vertex (PV). Leptons are required to be isolated and to have $\eta$ between 2.0 and 4.5, while for hadrons $\eta$ is required to be between 2.25 and 3.75. The combined invariant mass of the track pair must be greater than $20 \mathrm{GeV}$. In addition, for the di-muon final state the $p_{T}$ asymmetry must be above 0.3 , while in order to suppress the direct decays of $Z$ bosons into two muons, the muon pairs with invariant mass between 80 and $120 \mathrm{GeV}$ are excluded. Six background components have been considered: (i) $Z \rightarrow \tau \tau$, (ii) hadronic processes (QCD): leptonic $b$ - or $c$-hadron decay or misidentified hadron, (iii) electroweak processes, where one decay product candidate originates from a $W$ or $Z$ boson and the other comes from the underlying event, (iv) $t \bar{t}$, (v) $W W$ and (vi) $Z \rightarrow l l$, where $l l$ indicates electrons or muons. For the $Z \rightarrow \tau \tau, Z \rightarrow l l$ and QCD backgrounds both invariant mass distribution and normalization have been determined from data. In the case of QCD backgrounds same-sign leptons have been used. Small contributions from $t \bar{t}$ and $W W$ production have been taken from simulation.

The SM cross section has been taken from [8], while in the case of MSSM, the scenario with maximum Higgs boson mass has been considered, where the cross sections and efficiencies have 
been taken from gluon-fusion and associated $b \bar{b}$ production mechanisms. The upper limits for both model independent and MSSM Higgs boson production have been calculated using the method in [9] at $\mathrm{CL}_{S}=95 \%$. The upper limit on the cross-section times branching fraction of a model independent Higgs boson decaying to two tau leptons with $2.0<\eta<4.5$ is shown in Fig. 1(left), while the upper limit on $\tan \beta$ for the production of neutral MSSM Higgs bosons, as a function of the CP-odd Higgs boson mass, $M_{A^{0}}$, is plotted in Fig. 1(right).
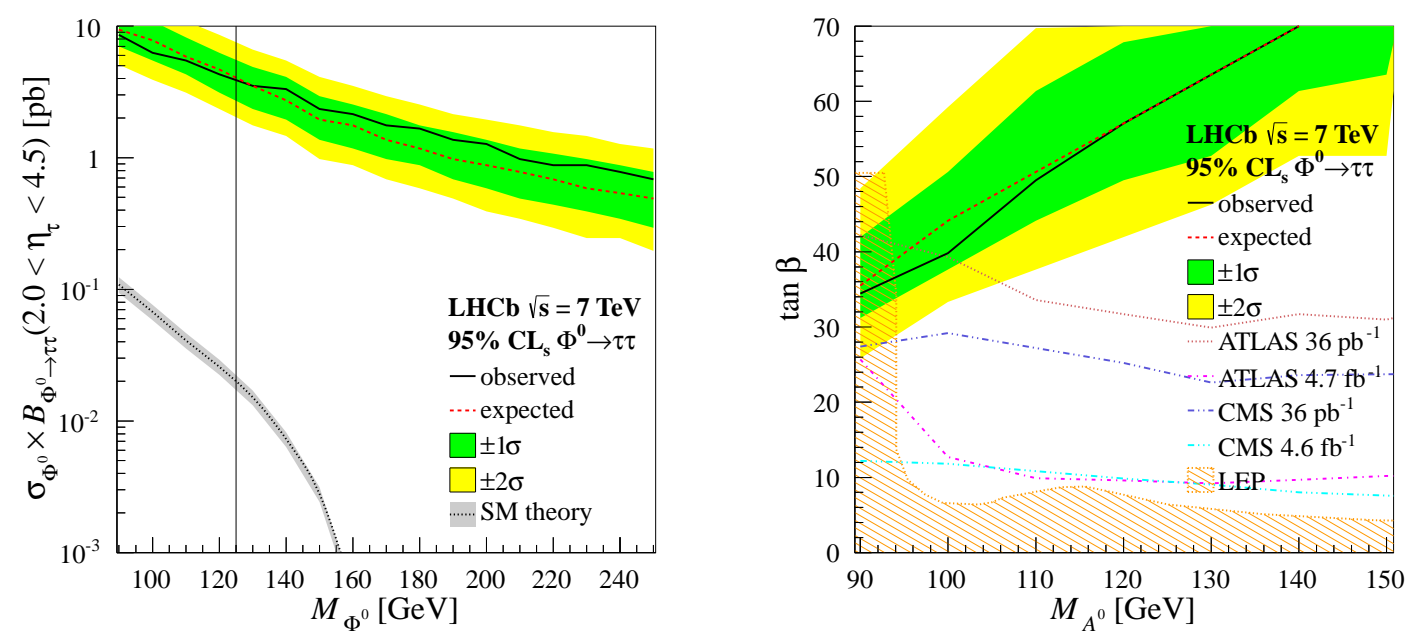

Figure 1: (Left) Model independent combined limit on cross-section times the branching fraction for a Higgs $\rightarrow \tau^{+} \tau^{-}$at $95 \%$ CLs as a function of the Higgs boson mass $M_{\Phi^{0}}$. (Right) The combined MSSM 95\% CLs upper limit on $\tan \beta$ as a function of $M_{A^{0}}$, compared to ATLAS (dotted maroon and dot-dashed magenta), CMS (dot-dot-dashed blue and dot-dot-dot-dashed cyan), and LEP (hatched orange) results.

\section{Search for Higgs-like bosons decaying into long-lived exotic particles}

The analysis has been performed using data at $\sqrt{s}=7 \mathrm{TeV}$ collected during 2010, corresponding to an integrated luminosity of $35.8 \mathrm{fb}^{-1}$ [10]. Hidden Valley models (HV) [4] as well as supergravity models with a barion number violation (BV) [5] predict the Higgs boson to decay into the combinations of massive long-lived particles (LLP) which may decay into $b \bar{b}$ pairs (HV) or three quarks (BV). Therefore, the analysis is based on secondary vertices displaced from the PV and the beam axis. At least one PV with radial distance to the beam axis below $0.3 \mathrm{~mm}$ and track multiplicity greater than ten is required, together with two LLP candidate vertices, each having at least six tracks and an invariant mass larger than $6 \mathrm{GeV}$. Since the crucial source of background comes from particles that have interacted with the material of the VELO, LLP candidate vertices are required to be in a fiducial volume defined to exclude VELO sensors and RF shield. In absence of radiative effects the two LLP's are expected to be almost back-to-back in the transverse plane, thus only the pairs of LLP candidates with $\Delta \phi>2.8 \mathrm{rad}$ are combined to form a parent (Higgs) candidate. 
The shapes of the distributions of single LLP candidates and of the combinations of two LLP's, as well as the yields, are compatible with the background coming from the inclusive $b \bar{b}$ events. The main systematic uncertainty comes from the inclusive displaced vertex reconstruction and the trigger efficiency, estimated by comparing the number of $b \bar{b}$ events with a large flight path in data and simulation. After the final selection, no event survives in the data. The upper limit on the cross section at 95\% CL using Feldman-Cousins method [11] have been calculated within the BV model in the sensitivity range covering long-lived particle lifetimes from 3 to $25 \mathrm{ps}$, masses between 30 and $55 \mathrm{GeV}$, and parent Higgs masses of 100-125 GeV. Higgs detection efficiencies have been taken from simulation. Preliminary upper limits on the cross section for the production of a Higgs boson decaying into LLP's, as a function of the LLP and Higgs masses for a fixed LLP lifetime are summarized in Table 1.

Table 1: 95\% CL upper limits on the cross section for the production of a Higgs boson, as a function of the LLP and Higgs mass $m_{h^{0}}$ for a LLP lifetime of $10 \mathrm{ps.} \mathrm{Cross} \mathrm{sections} \mathrm{are} \mathrm{given} \mathrm{in} \mathrm{pb.} m_{L L P}$ denotes the LLP mass.

\begin{tabular}{|c||c|c|c|c|c|}
\hline \multicolumn{1}{|c||}{} & \multicolumn{5}{c|}{$m_{L L P}\left(\mathrm{GeV} / \mathrm{c}^{2}\right)$} \\
\hline$m_{h^{0}}\left(\mathrm{GeV} / \mathrm{c}^{2}\right)$ & 30 & 35 & 40 & 48 & 55 \\
\hline \hline 100 & 101 & 58 & 44 & 58 & - \\
105 & 100 & 75 & 44 & 39 & - \\
110 & 132 & 75 & 56 & 34 & - \\
114 & 128 & 91 & 47 & 32 & 46 \\
120 & 148 & 93 & 58 & 34 & 31 \\
125 & 179 & 90 & 61 & 41 & 29 \\
\hline
\end{tabular}

\section{Measurement of $\sigma(\mathbf{b} \bar{b})$ and $\sigma(\mathbf{c} \bar{c})$ with inclusive final states}

The measurement has been performed using 2010 data at $\sqrt{s}=7 \mathrm{TeV}$, corresponding to an integrated luminosity of $35.8 \mathrm{fb}^{-1}$ [12]. The selection procedure relies on the reconstruction of inclusive secondary vertices originating from the $b$-hadron decays. This procedure selects, with lower efficiency, an inclusive sample of $c \bar{c}$ as well. Therefore, the $b \bar{b}$ and $c \bar{c}$ cross sections may be simultaneously measured. In the first step inclusive $b$ or $c$ candidates (defined as 'seeds') are identified through the secondary vertex reconstruction from two or three tracks likely to be a part of the decay products of a $b$ or $c$ hadron. In order to assign the reconstructed seeds to a PV, only events with exactly one reconstructed PV have been used, and the corresponding luminosity has been calculated to be $2.633 \mathrm{pb}^{-1}$. The $b$ hadron direction is approximated with a very good precision by the direction of the reconstructed seed, as it may be seen in Fig. 2, where simulated $b \bar{b}$ inclusive events have been used. Since pairs of seeds close in azimuthal angle originate usually from the same $b$ hadron, a merging procedure has been applied to recombine such candidates, if the invariant mass of all tracks forming the two seeds is below $5.5 \mathrm{GeV}$. The seed energy has been calibrated according to the energy of generated $b$-hadron. 
As the trigger efficiency as a function of the seed pseudorapidity is constant at $2.5<\eta<$ 4.0, this interval has been chosen as a fiducial region of the measurement. The total efficiency is the product of the trigger, offline preselection, seeding and final seed selection efficiencies, and it is estimated based on simulated $b \bar{b}$-inclusive and $c \bar{c}$-inclusive samples. It amounts to $(8.0 \pm$ $1.1) \times 10^{-4}$ for $b \bar{b}$ and to $(1.8 \pm 0.2) \times 10^{-5}$ for $c \bar{c}$. The contribution from light quarks is found to be negligible. The Monte Carlo based $b$-tagging efficiency has been verified using the data sample of $B^{0} \rightarrow D \pi$ exclusive mode. The opposite side $B$ is then considered to be representative of an inclusive $b \bar{b}$ sample. Contributions from $b \bar{b}$ and $c \bar{c}$ are determined from the fit to a Boosted Decision Tree Gradient (BDTG) [13] discriminant. The result of the fit to the data using the $b \bar{b}$ and $c \bar{c}$-inclusive templates is shown in Fig.3. The preliminary measurement of the cross section based on seed pairs with $p_{T}>5 \mathrm{GeV}$ and $2.5<\eta<4.0$ is as follows:

$$
\sigma^{b \bar{b}}=7.7 \pm 0.12(\text { stat }) \pm 0.84 \text { (syst) } \mu \mathrm{b}
$$

and

$$
\sigma^{c \bar{c}}=104.6 \pm 2.7 \text { (stat) } \pm 11.4 \text { (syst) } \mu \mathrm{b} .
$$

Extrapolating the $b \bar{b}$ cross section (4.1) to the full $\mathrm{LHCb}$ acceptance, the value of $\sigma^{b \bar{b}}=79.7 \pm$ 1.2 (stat) \pm 8.7 (syst) $\mu \mathrm{b}$ is obtained. It is compatible with the previous LHCb measurement based on exclusive decay channels [14]. The main systematic uncertainty comes from the size of the simulated sample used and the integrated luminosity.
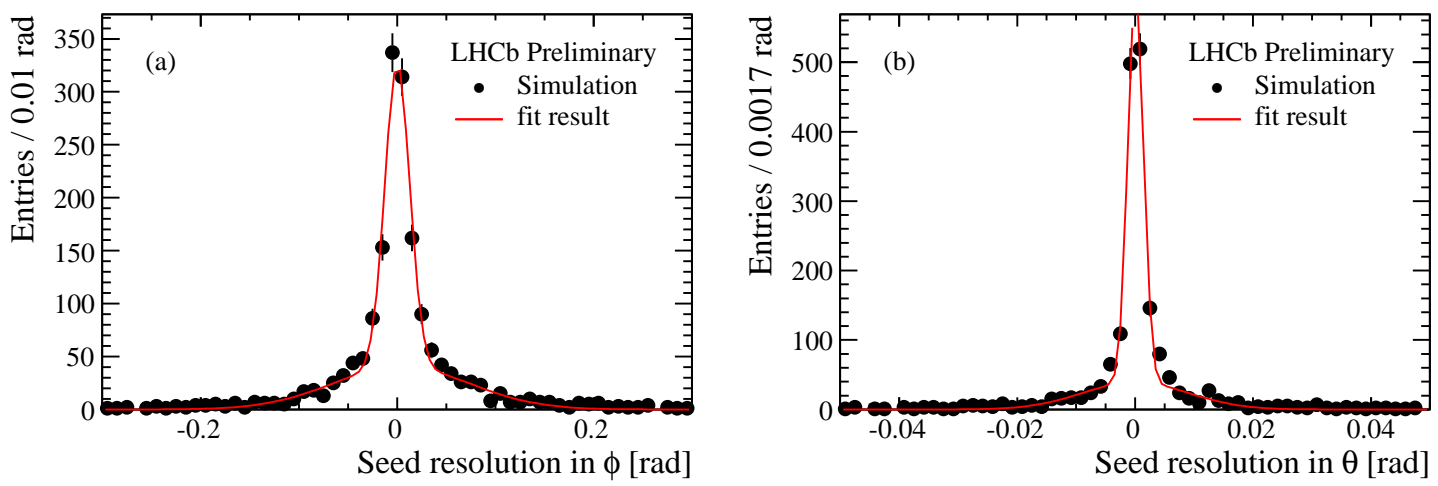

Figure 2: Resolution in (a) $\phi$ and (b) $\theta$ for the seed measured as described in the text for events with one $b$ hadron and one reconstructed seed.

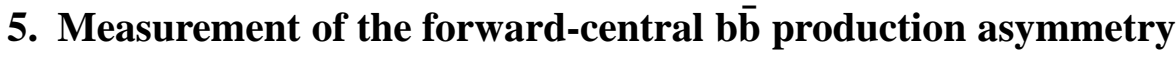

The dataset used for this analysis has been taken at a centre-of-mass energy of $7 \mathrm{TeV}$, which corresponds to an integrated luminosity of about $1.0 \mathrm{fb}^{-1}$ [15]. Events are selected based on high $p_{T}$ jet pairs (jet $p_{T}>15 \mathrm{GeV}$ ), where jets are in the $\eta$ region between 2 and 5 and are approximately back-to-back $\left(\Delta \phi_{j j}>2.5 \mathrm{rad}\right)$. The jet reconstruction uses the anti- $k_{T}$ algorithm with a radius parameter $R=0.5$, where the input is a collection of tracking and calorimeter information used to build unique particle objects. The jet energy correction procedure accounts for out-of-acceptance 


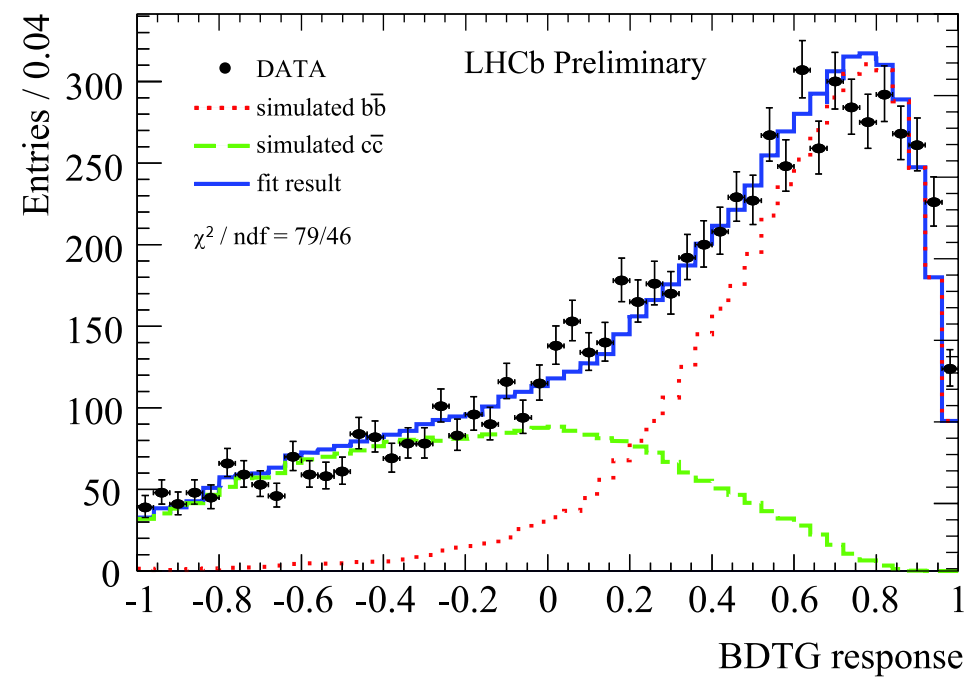

Figure 3: Response of the BDTG (defined in the text) for data (black dots) fitted with shapes evaluated from simulated $b \bar{b}$ (red dotted line) and $c \bar{c}$ (green dashed line) events. The fit result is indicated by the solid blue line.

particles, calorimeter response, noise and pile-up, and is derived using simulation. It is validated by comparing the $p_{T}$ balance of events with a $Z$ and a jet in data and simulation. The jet energies are corrected and the resolution on the di-jet mass is (15-20)\%. Measuring the forward-central asymmetry $\left(A_{F C}^{b \bar{b}}\right)$ requires that two jets are $b$-tagged, based on the identification of 2-, 3- or 4-track vertices significantly displaced from the PV and compatible with $b$-hadron decays. In order to establish if the jet originates from the $b$ - or $\bar{b}$-quark hadronization, one of the jets is required to contain a displaced track identified as a muon. The purity of such identification is validated by using data samples, where one $b$-quark is found from fully reconstructed $B \rightarrow J / \psi K$ or $B \rightarrow D \pi$ decays.

The $\Delta y$ distributions for all events and, separately, only for those with a measured $M_{b \bar{b}}>$ $100 \mathrm{GeV}$ are shown in Fig. 4. The resolution in $\Delta y$ of about 0.1 has negligible effect on the final $A_{F C}^{b \bar{b}}$. The asymmetry is measured separately for the two polarities of the LHCb dipole magnet and the arithmetic mean of these two measurements is calculated. The preliminary result on the asymmetries corrected for the flavor-tagging impurity is:

$$
A_{F C}^{b \bar{b}}=(0.5 \pm 0.5 \text { (stat) } \pm 0.5 \text { (syst) }) \%
$$

and

$$
A_{F C}^{b \bar{b}}\left(M_{b \bar{b}}>100 G e V\right)=(4.3 \pm 1.7(\text { stat }) \pm 2.4(\text { syst })) \%,
$$

where systematic errors include the uncertainties on the flavor-tagging purity and possible detector asymmetry. It should be stressed that $M_{b \bar{b}}$ is not corrected for detector resolution (not unfolded).

\section{References}

[1] CMS collaboration, Phys. Lett. B710 (2012) 26, 

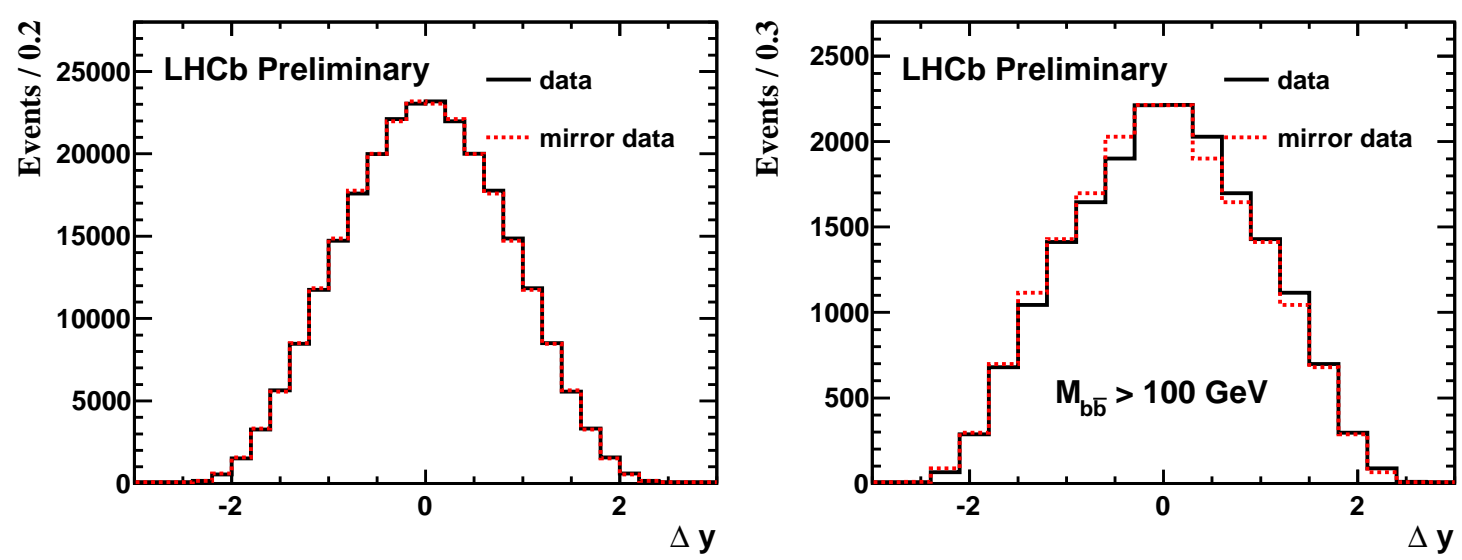

Figure 4: Observed $\Delta y$ distributions for (left) all events and (right) those with measured $M_{b \bar{b}}>100 \mathrm{GeV}$. Dashed distribution corresponds to $\Delta y \rightarrow-\Delta y$.

ATLAS collaboration, Phys. Rev. D86 (2012) 032003.

[2] LHCb collaboration, A. A. Alves Jr. et al., The LHCb detector at the LHC, JINST 3 (2008) S08005.

[3] S. P. Martin, A Supersymmetry Primer, ed. G.L. Kane: Perspectives on supersymmetry II, (1997) 1.

[4] M.J. Strasser, K.M. Zurek, Echoes of a hidden valley at hadron colliders, Phys. Lett. B651 (2007) 374.

[5] L. M. Carpenter, D. E. Kaplan and E. J. Rhee, Reduced Fine-Tuning in Supersymmetry with R-parity Violation, Phys. Rev. Lett. 99 (2007) 211801.

[6] D0 collaboration, V. Abazov et al., Measurement of the top quark forward-backward production asymmetry and its dependence on event kinematic properties, Phys. Rev. D84 (2011) 112005.

[7] LHCb collaboration, R. Aaij et al., Limits on neutral Higgs boson production in the forward region in pp collisions at $\sqrt{s}=7 \mathrm{TeV}$, JHEP 05 (2013) 132.

[8] D. de Florian, M. Grazzini, Higgs production through gluon fusion: Updated cross sections at the Tevatron and the LHC, Phys. Lett. B674 (2009) 291.

[9] A. L. Read, Presentation of search results: The CL(s) technique, J. Phys. G28 (2002) 2693.

[10] LHCb collaboration, Search for Higgs-like bosons decaying into long-lived exotic particles, LHCb-CONF-2012-014.

[11] G. J. Feldman, R. D. Cousins, A unified approach to the classical statistical analysis of small signals, Phys. Rev. D57 (1998) 3873.

[12] LHCb collaboration, Measurement of $\sigma(b \bar{b})$ with inclusive final states, LHCb-CONF-2013-002.

[13] L. Breiman, J. H. Friedman, R. A. Olshen, and C. J. Stone, Classification and regression trees, Wadsworth international group, Belmont, California, USA, 1984.

[14] LHCb collaboration, Measurement of $\sigma(p p \rightarrow b \bar{b} X) a t \sqrt{s}=7 \mathrm{TeV}$ in the forward region, Phys. Lett. B694 (2010) 209.

[15] LHCb collaboration, Measurement of the forward-central $b \bar{b}$ production asymmetry, LHCb-CONF-2013-001. 